\title{
FUMO DURANTE A GESTAÇÃO: ASPECTOS EDUCATIVOS DE DE UM PROBLEMA COMPORTAMENTAL
}

\author{
Nelly Martins Ferreira Candeias *
}

RSPUB9/468

CANDEIAS, N. M. F. Fumo durante a gestaçäo: aspectos educativos de um problema comportamental. Rev. Saúde públ., S. Paulo, 13:244-53, 1979.

RESUmo: Descreve-se o hábito de fumar de mulheres internadas no Serviço de Obstetricia de um hospital do municipio de São Paulo, Brasil, assim como conhecimentos a respeito dos efeitos do fumo durante a gestação e orientação recebida durante esse periodo. Apresentam-se algumas implicações para a educação em saúde, considerando-se diferentes niveis de programas anti-fumo.

Unitermos: Fumo. Gravidez. Educaşão em saúde pública, programas.

O tabagismo representa um sério risco para o bem-estar individual. Seus efe.tos passaram despercebidos, por muito tempo, por se manifestarem em geral a longo prazo, variando aproximadamente de 20 a 40 anos após o início deste hábito aparentemente inócuo. Ao contrário do que sucede nos casos decorrentes da ingestão de drogas, não se registram intoxicações agudas, nem conseqüências espetaculares e imediatas. São tardias as conseqüências da exposição ao risco do fumo, manifestando-se através de moléstias pulmonares crônicas, alterações cardio-vasculares, câncer de pulmão e da laringe, entre outros agravos.

Há um grupo populacional, entretanto, que sofre efeitos tão imediatos e detectáveis, que justifica a inclusão do hábito de fumar entre os fatores a considerar em sistemas de avaliação do risco gravídico:
0 grupo de gestantes. Apesar das consequiências imediatas que o fumo tem para a saúde materno-fetal, não existe no Brasil estudo que apresente dados a respeito do hábito de fumar durante a gestação, assin como a respeito de conhecimentos sobre os efeitos do fumo entre mulheres em idade fértil e de baixo nivel sócio-econômico. Também não existem dados referentes à orientação recebida durante o ciclo gravidico-puerperal, razão esta que levou ao desenvolvimento do presente estudo, cujos objetivos são:

- descrever o hábito de fumar numa população de mulheres de baixo nivel sócio-econômico;

- analisar o nivel de conhecimento das entrevistadas no que concerne aos efeitos do fumo para a saúde maternoinfantil;

* Do Departamento de Prática de Saúde Pública da Faculdade de Saúde Pública da USP Av. Dr. Arnaldo, 715 - 01255 - São Paulo, SP - Brasil. 
CANDEIAS, N. M. F. Fumo durante a gestação: aspectos educativos de um problema comportamental. Rev. Saúde puibl,. S. Paulo, 13:244-53. 1979.

- verificar se as gestantes receberam orientação a respeito de como proceder em relação ao hábito de fumar durante a gestaçāo;

- caracterizar o papel da educação em saúde no que respeita à educação anti-fumo.

\section{MATERIAL E MÉTODOS}

Estudo sobre educação em saúde aplicada à prevenção do risco gravídico pré-natal e interconcepcional (Candeias ${ }^{5}$, 1979) permitiu a formação de um banco de dados, parte dos quais foram coletados com vistas à realização de trabalhos subsequentes.

A população desse estudo foi constituida por 404 mulheres internadas em um serviço de obstetricia de um hospital de São Paulo, no periodo de abril a setembro de 1975 . Após receberem alta médica, as puérperas foram inquiridas consecutivamente, dando origem à informação básica do presente trabalho. Os dados foram processados por computador.

Este estudo descreve dados referentes ao hábito de fumar. As perguntas incluidas no formulário, sobre esse aspecto foram:

- Você fuma? (Se a resposta for sim) Você continuou fumando o mesmo número de cigarros durante a gravidez?

- Algumas pessoas dizem que fumar durante a gravidez faz mal à saúde da mãe e do nenê. Outras pessoas dizem que fumar durante a gravidez não faz mal, nem para a saúde da mãe e nem para a saúde do nenê. $O$ que você acha: fumar faz mal ou não faz mal para a saúde da mãe? E para a saúde do nenê?

- Alguém disse a você para não fumar ou para fumar menos durante a gravidez? (Se for o caso) Quem?

\section{RESULTADOS E DISCUSSĀO}

Conforme a Figura 1, verificou-se que $27,9 \%$ das entrevistadas fumavam, $4,5 \%$ haviam deixado de fumar e $67,6 \%$ não fumavam.

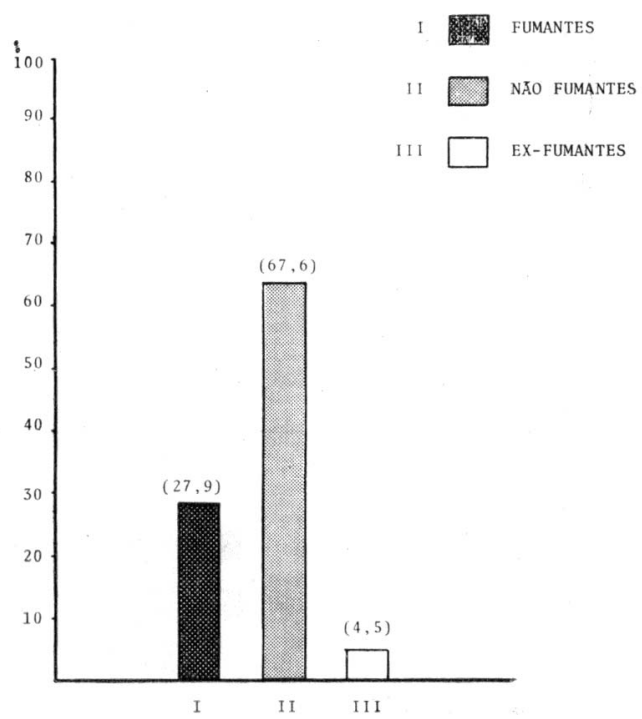

Fig. 1 - Comportamento de gestantes em relação ao hábito de fumar.

A Tabela 1 permite observar a frequiencia absoluta e relativa do comportamento referente ao tabagismo segundo grupo etário.

Nessa Tabela, chama a atenção o fato de $30,8 \%$ de jovens com 20 anos ou menos fumarem regularmente; no que concerne a outros grupos etários, encontrou-se $32,7 \%$ de mulheres com 21 a 24 anos, $25,0 \%$ no grupo com 25 a 29 anos, $31,4 \%$ no grupo com 30 a 34 anos, $17,5 \%$ no grupo com 35 a 39 anos e $19,0 \%$ no grupo com 40 anos ou mais que têm o hábito de fumar.

Houve interesse em verificar o conhecimento da população amostral a respeito dos efeitos do fumo para a saúde materno. -fetal.

De acordo com a Fig. 2, 19,0\% (76 mulheres) afirmam que fumar durante a gestação prejudica apenas o feto, $4,5 \%$ 
CANDEIAS. N. M. F. Fumo durante a gestação: aspectos educatiros de um problema comportamental. Rev. Saúde públ., S. Paulo, 13:244-53, 1979.

T A B E L A 1

Hábito de fumar de puérperas internadas em Serviço de Obstetrícia, de um Hospital de São Paulo, segundo faixa etária.

\begin{tabular}{|c|c|c|c|c|c|c|c|c|c|c|c|}
\hline \multirow{3}{*}{ I dad } & & \multicolumn{10}{|c|}{ Hábito de fumar } \\
\hline & & \multicolumn{2}{|c|}{ Fumantes } & \multicolumn{2}{|c|}{$\begin{array}{c}\text { Não } \\
\text { fumantes }\end{array}$} & \multicolumn{2}{|c|}{$\begin{array}{c}\text { Abandonaram } \\
\text { o fumo }\end{array}$} & \multicolumn{2}{|c|}{ Diminuiram } & \multicolumn{2}{|c|}{ Totil } \\
\hline & & $N "$ & $\%$ & No & & $N^{*}$ & $\%$ & No & $\%$ & $N$ & $\%$ \\
\hline 15 & 211 & 10 & 14.7 & 42 & $61 . x$ & 5 & 7.4 & 11 & 16.1 & $6 x$ & $10 \% 1.0$ \\
\hline$\because 1$ & 24 & $1: 3$ & 12.9 & (i) & 60.4 & 7 & 6.9 & 241 & $19, n$ & 1011 & 1011,11 \\
\hline 25 & 29 & $1: ;$ & 13.5 & 711 & 72.9 & 2 & 2.1 & 11 & 11.5 & 96 & $11+1,11$ \\
\hline$: 31$ & 34 & 10 & 19.6 & 33 & 64.7 & 2 & $\therefore .9$ & b & 11,4 & 51 & 100.11 \\
\hline$: 85-$ & 34 & 2 & 5.11 & 32 & 811.0 & 1 & 2.5 & 5 & 12,5 & 411 & 100,0 \\
\hline 111011 & t & $: ;$ & 14.2 & 17 & 81.0 & & & 1 & 4.6 & 21 & 11110.11 \\
\hline
\end{tabular}

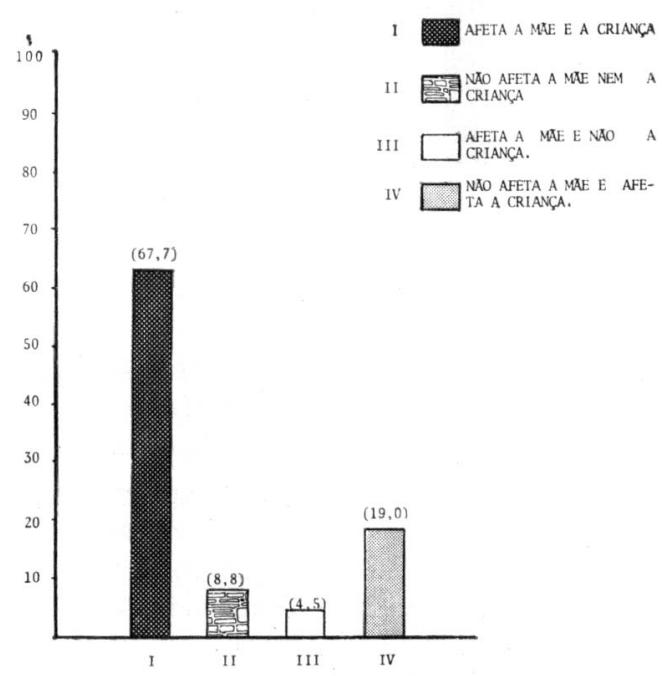

Fig. 2 - Opinião de gestantes a respelto dos pfeitos do fumo parat an uniade materno-infantil.

(18 mulheres) acham que prejudica apenas a mãe, $8,8 \%$ (35 mulheres) que não afeta nem a mãe e nem a criança, enquanto que $67,7 \%$ (270 mulheres) consideram que, ao contrário, afeta a diade materno-fetal. Chama a atenção o fato de $32,3 \%$ das inquiri- das terem unl conhecimento incorreto a respeito dos efeitos do fumo para a unidade materno-fetal.

A Tabela 2 complementa estes dados na medida em que permite observar a opiniāo das entrevistadas segundo faixa etária.

No que se lefere às conseyuências do fumo para a saúde humana, cumpre mencionar que estudos prospectivos realizados em diferentes áreas geográficas têm chamado a atenção para aspectos significativos do ponto de vista da saúde publica. Cabe mencionar, por sua importancia, o estudo de Hammond ${ }^{18}$, patrocinado pela Sociedade Americana contra o Câncer, que abrangeu 25 estados e 1.048.138 individuos, cujos resultados permitiram verificar que: a mortalidade geral é maior entre fumantes; a mortalidade aumenta en função do número de cigarros consumidos, do periodo de tempo decorrido e da quantidade de fumo inalado. Também se verificou que a proporção de indivíduos com câncer de pulmão, de boca, de laringe e esôfago, assim como com bronquites e enfermidades pulmonates e circulatórias é maior do que entre indivíduos não fumantes. Não basta, entretanto, mencionar apenas as conseqüèn- 
CANDEIAS, N. M. F. Fumo durante a gestação: aspectos educativos de um problema comportamental. Rev. Saúde públ., S. Paulo, 13:244-53, 1979.

TABELA 2

Opiniāo a respeito do fumo de um grupo de puérperas internadas em Serviço de Obstetrícia de um Hospital de São Paulo, segundo a faixa etária.

\begin{tabular}{|c|c|c|c|c|c|c|c|c|c|c|c|c|}
\hline \multirow{2}{*}{\multicolumn{3}{|c|}{ Idade }} & \multicolumn{2}{|c|}{$\begin{array}{l}\text { Afeta a mãe/ } \\
\text { Afeta a criança }\end{array}$} & \multicolumn{2}{|c|}{$\begin{array}{c}\text { Não afeta a mãe/ } \\
\text { Não afeta } \\
\text { a criança }\end{array}$} & \multicolumn{2}{|c|}{$\begin{array}{l}\text { Afeta a mãe/ } \\
\text { Não afeta } \\
\text { a criança }\end{array}$} & \multicolumn{2}{|c|}{$\begin{array}{l}\text { Não afeta a mãe/ } \\
\text { Afeta a criança }\end{array}$} & \multicolumn{2}{|c|}{ Total } \\
\hline & & & No & $\%$ & No & $\%$ & No & $\%$ & No & $\%$ & Ne & $\%$ \\
\hline 15 & - & 20 & 48 & 64,0 & 6 & 8,0 & 3 & 4,0 & 18 & 24,0 & 75 & 100,0 \\
\hline 21 & - & 24 & 65 & 61.9 & 11 & 10.5 & 5 & 4,8 & 24 & 22,8 & 105 & 100,0 \\
\hline 25 & - & 29 & 711 & 68,6 & 7 & 6.9 & i & 5,9 & 19 & 18,6 & 102 & 100,0 \\
\hline 30 & - & 34 & 40 & 71,4 & 4 & 7,2 & 1 & 1,8 & 11 & 19,6 & 56 & 100,0 \\
\hline 35 & - & 39 & 31 & 79.5 & 4 & 10,3 & 2 & 5,1 & 2 & 5,1 & 39 & 100,0 \\
\hline 40 & ou & + & 16 & 72,7 & 3 & 13,6 & 1 & 4,5 & 2 & 9,2 & 22 & 100,0 \\
\hline
\end{tabular}

cias que o fumo tem, a longo prazo, para os adultos considerados isoladamente. E preciso analisar, também, os efeitos imediatos que este hábito tem para a saúde da unidade materno-fetal.

Uma das primeiras referências aos efeitos negativos do fumo durante a gestação, no que concerne ao desenvolvimento do feto, foi apresentada por Simpson 16, em 1957. Relatou este autor que a incidência de recém-nascidos de baixo peso $(2500 \mathrm{~g}$ ou menos) mostrava-se significantemente maior entre mulheres fumantes do que entre mulheres não-fumantes.

Em 1959, Lowe ${ }^{14}$, ao estudar uma população amostral constituída por 2.042 mulheres, que deram à luz em Birmingham, na Inglaterra, mostrou que o peso médio dos recém-nascidos de mães fumantes era cerca de $170 \mathrm{~g}$ inferior ao peso médio de recém-nascidos de mães não-fumantes. Embora neste estudo não tenha havido associação entre o hábito de fumar e complicações da gravidez, a proporção de gestações que culminaram com partos induzidos cirurgicamente, foi significantemente menor entre mulheres não-fumantes.

Mencionado por Goldstein 10, Yerushalmy observou que o baixo peso ao nascer, assim como o aumento de óbitos no periodo perinatal, deviam-se não apenas ao ato de fumar, mas ao fato de que o tipo de mulher que fumava coincidia com o tipo de mulher que apresentava, também, o risco de gerar crianças com baixo peso, em decorrência de outros fatores. É esta a razão pela qual se têm desenvolvido estudos sobre o hábito de fumar, que consideram a influência de fatores intermediários, como classe social, idade e paridade. Estes estudos permitiram observar que, de modo geral, a proporção de casos de crianças com baixo peso, assim como de óbitos no período perinatal é maior entre mulheres fumantes. Aceita-se que, crianças de mães fumantes pesem cerca de 180 a $230 \mathrm{~g}$ menos do que as demais crianças, apresentando, portanto, maior risco de mortalidade perinatal. ${ }^{1}$

$O$ baixo peso ao nascer não representa, entretanto, o único efeito pernicioso do hábito de fumar durante o periodo da gestação. Villumsen, mencionado por Goldstein 10 , ao entrevistar 1.323 mulheres, em 1962 , observou que aquelas que fumavam mais do que três cigarros por dia durante a gestaçāo, apresentavam uma taxa de prematuridade significantemente maior $(21,3 \%)$ do que mulheres que fumavam 
CANDEIAS, N. M. F. Fumo durante a gestação: aspectos educativos de um problema comportamental. Rev. Saúde públ, S. Paulo, 13:244-53, 1979.

três ou menos cigarros $(15,7 \%)$. Também em 1962, Herriot e col. ${ }^{13}$, controlando a variável nível sócio-econômico, relataram ser mais frequiente a prematuridade entre os recém-nascidos de mulheres fumantes. Butler e col.4, em 1972, referiram proporções significantemente distintas de óbitos perinatais entre mulheres não-fumantes, fumantes intermediárias ( 1 a 4 cigarros diários) e mulheres que fumavam cinco ou mais cigarros por dia. Complementando, Duffus e Macgillivray ${ }^{9}$ chamaram a atenção para o risco de pré-eclampsia durante o período da gestação. Como efeito a longo prazo, Butler e Goldstein " fizeram referência a problemas de natureza intelectual em crianças com 7 e com 11 anos, filhos de mulheres que fumaram 10 ou mais cigarros por dia durante a gravidez, afirmação esta não confirmada por Hardy e Mellits. ${ }^{12}$

Não se conhecem exatamente os mecanismos responsáveis pelos efeitos do fumo sobre o peso do recém-nascido. Poder-seia imaginar que o fumo afeta o apetite materno, reduzindo o peso da gestante e, em decorrência disto, interferindo no peso do recém-nascido. Este não tem sido considerado, no entanto, o principal fator responsável pela redução do peso ao nascer, muito embora alguns autores tenham decidido reestudar este fato. ${ }^{8} \mathrm{~A}$ vasoconstrição, provocada pelo fumo, poderia ter também um efeito apreciável na nutrição fetal como conseqüência de uma diminuição do fornecimento do sangue que alcança os espaços intervilosos, reduzindo assim o teor de substâncias nutritivas e causando uma retenção de catabolitos, como sugere Goldstein. ${ }^{10}$ De início, julgou-se que o efeito vasoconstritor da nicotina fosse o fator mais importante; atualmente, considera-se o monóxido de carbono do cigarro fator ainda mais comprometedor, já que a hemoglobina do sangue tem maior afinidade pelo monóxido de carbono do que pelo oxigênio. ${ }^{7}$ Tem-se mencionado que uma alta concentração daquele tende a reduzir a capacidade de transportar o oxigênio do sangue, atuando como um agente teratogênico que aumenta o risco de mal desenvolvimento e de morte fetal. Complementando, tem-se referido o conteúdo de cianeto do cigarro como elemento nocivo ao crescimento fetal em decorrência de seus efeitos sobre o metabolismo da vitamina $B_{12}$ da idade materno-fetal. ${ }^{1 ;}$

Estas considerações justificam a ênfase que se deve conceder a conteúdos programáticos anti-fumo, dirigidos, no presente caso, a mulheres em idade fértil.

A par da opinião a respeito das conseqüências do fumo para a unidade materno-fetal, procurou-se também verificar até que ponto a população amostral recebeu ou não orientação a respeito de como proceder em relação ao hábito de fumar durante o ciclo gravídico.

De acordo com a Fig. 3, uma proporção bastante elevada de mulheres fumantes, $37,0 \%$, não receberam nenhuma informação a respeito de como proceder durante a gestação, sendo que $22,8 \%$ foram orientadas por médicos e $1,3 \%$ por enfermeiras;

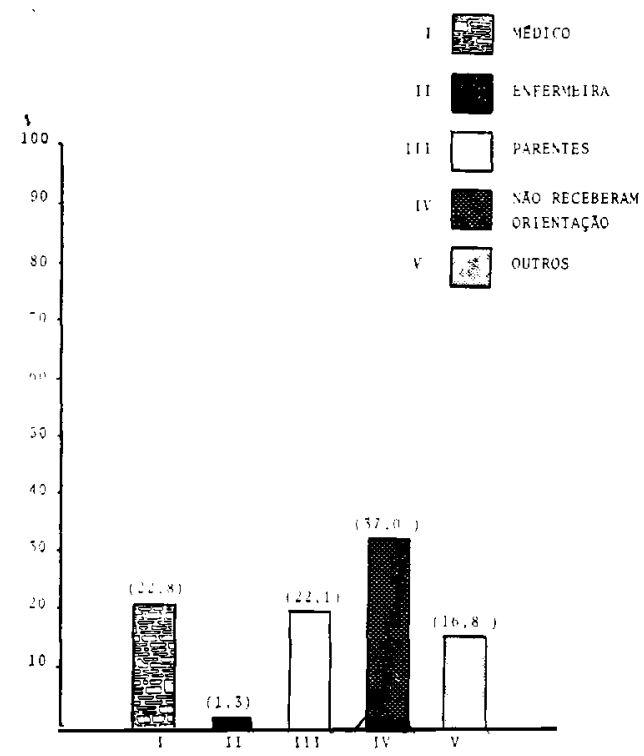

Fig. 3 - Orientação recebida pelas fumantes a respeito de como proceder durante a gestação. 
CANDEIAS, N. M. F. Fumo durante a gestação: aspectos educativos de um problema comportamental. Rer. Saúde públ., S. Paulo, 13:244-5?, 1979.

das restantes, $21,1 \%$ foram orientadas por parentes e $16,8 \%$ por outros que não os grupos já referidos.

A Tabela 3 complementa estas observações. Cumpre ressaltar que em todas as faixas etárias, com exceção do grupo com 21 a 24 anos, recaem os valores modais sobre mulheres que não receberam nenhuma orientação sobre os efeitos do fumo para a saúde materno-fetal.

Estes dados assumem particular significado por se referirem a uma população de baixo nivel sócio-econômico.* O hábito de fumar não se distribui igualmente entre a população feminina. Segundo Baric e col. ${ }^{1}$, a variavel que se tem mostrado mais intimamente associada com o hábito de fumar durante a gravidez é a classe social. Ao realizar a Pesquisa Britânica de Mortalidade Perinatal, Butler e Alberman ? observaram que a proporção de fumantes variava significantemente nas diferentes estratificaçöes sociais: nas classes de nivel sócio-econômico mais elevado apenas $21,0 \%$ de gestantes fumavam, enquanto que nas classes de menor renda a percentagem alcançava o valor de $35,9 \%$. Para Baric e col. ${ }^{1}$, isto se deve ao fato de haver um maior numero de fumantes entre mulheres de baixo nivel sócio-econômico, representando estas, por outro lado, o grupo menos propenso a abandonar o hábito de fumar durante a gestaçāo. Tais consideraçōes são significativas para educadores de saude pública, visto que mulheres de baixo nivel sócio-economico apresentam maior risco de darem à luz crianças com baixo peso. Lamentavelmente, constitui este o grupo para quem, por um lado, seria mais importante abandonar o hábito de fumar durante a gestaçāo e, por outro menos suscetivel à aceitação de atividades relacionadas à educaçāo em saúde. Mencionados por Baric e col. ${ }^{2}$ os estudo de Lowe, Butler e col. e Schwartz e col afirmam ser no último período da gestação que o fumo mais prejudica o crescimento fetal. De acordo com dados desses autores, o peso ao nascer das crianças de mães que deixaram de fumar na segunda metade da gestaçāo não se distanciava sensivelmente do peso ao nascer de crianças de mulheres não-funantes. Criam-se assim condições favoráveis para educandos e educadores, por ter a gestante tempo suficiente para abandonar o fumo ao receber uma orientação correta no período inicial da gravidez durante o atendimento pré-natal. Chama-se a atenção para o fato de que, mesmo que esta não consiga parar de fumar durante a gestação, ainda se tem tempo suficiente para aconselhá-la a reduzir o número de cigarros fumados, tendo em vista os benefícios dai decorrentes.

O hábito do tabagismo representa um problema comportamental que, analisado do ponto de vista da saúde publica, contribui de modo bastante significativo para a morbidade da sociedade moderna. Pode-se atribuir o agravamento deste problema à expansão das atividades internacionais que utilizam amplamente os meios de comunicação de massa para promoção de seus produtos, à hipertrofia da medicina curativa em detrimento da medicina preventiva e, finalmente, à incapacidade que as sociedades ocidentais têm de utilizar mecanismos de caráter politico-social para controlar problemas de saúde através de medidas preventivas.

Torna-se evidente que um problema de saúde de natureza comportamental e de etiologia multifatorial, não pode ser controlado a não ser por programas que focalizem, concomitantemente, diferentes áreas: em primeiro lugar, é preciso considerar a educação médica já que os currícula de escolas médicas não enfatizam suficientemente os efeitos do fumo para a saúde dos individuos; em segundo lugar, deve-se

* A descrição do nível sócio-económico da população amostral encontra-se no estudo intitulado "Educação em Saude na prevenção do risco gravidico pré-natal e interconcepcional", de CANDEIAS. 
CANDEIAS, N. M. F. Fumo durante a gestaçăo: aspectos educativos de um problema comportamental. Rev. Saúde públ., S. Paulo, 13:244-53. 1979.

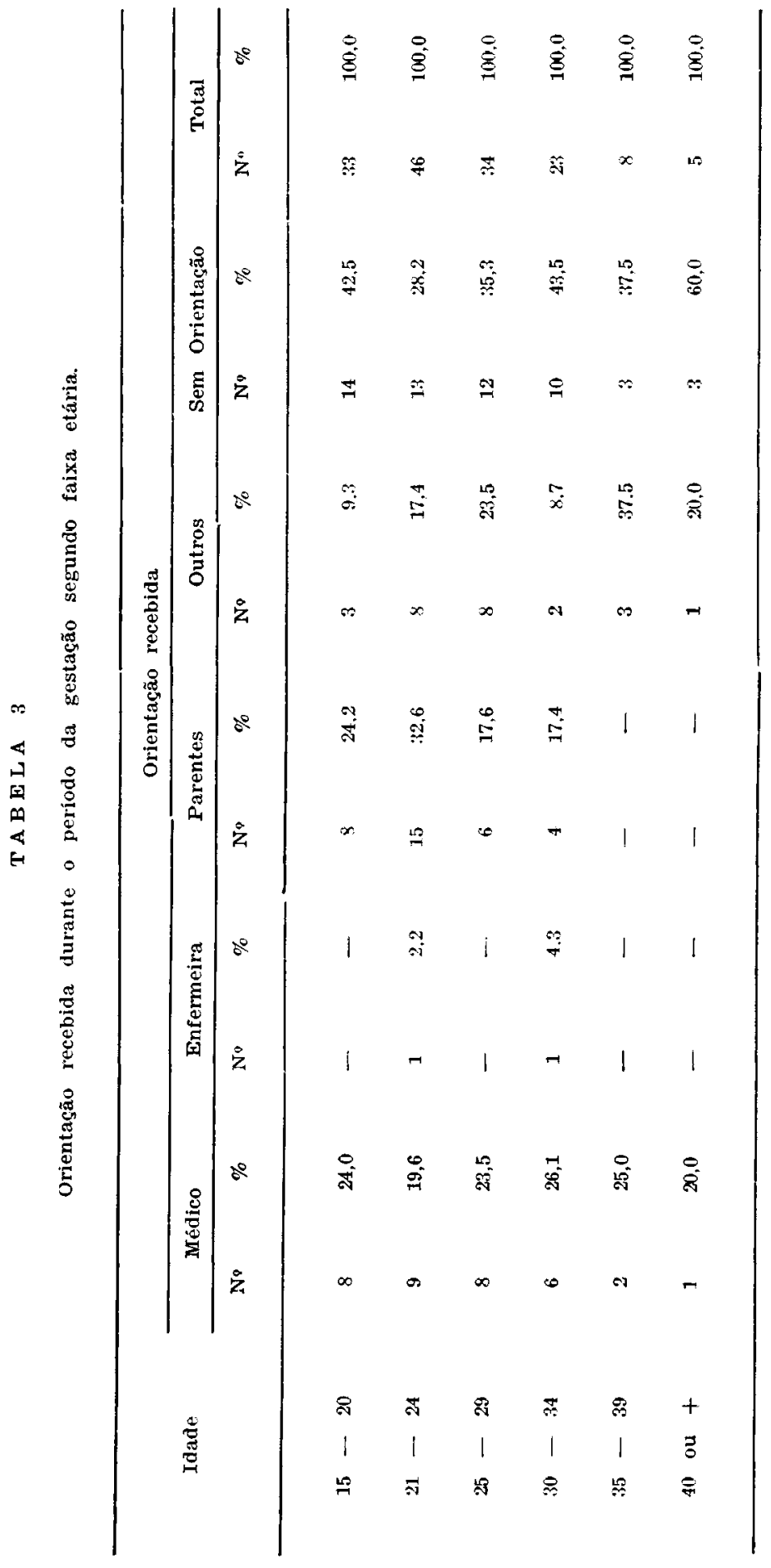


CANDEIAS, N. M. F. Fumo durante a gestação: aspectos eduratiros de um prohlema comportamental, Rev. Saude públ., S. Paulo, 13:244-5:3, 1979.

considerat a saúde na escola. A escola, por reunir grande número de crianças, representa o ponto de partida ideal para se iniciar a educação anti-fumo.19

No que concerne a programas anti-fumo dirigidos especificamente a crianças, cumpre desenvolver dois pontos:

(a) um programa nacional integrando todos os segmentos sociais de modo que as crianças vivam numa atmosfera anti-fumo, dos pontos de vista físico, social e psicológico;

(b) programas específicos com vistas a alcançar grupos de crianças com maior risco de contrair o hábito.

Considerando-se o fato de que, no presente estudo, a maioria das mulheres entrevistadas tiveram acesso apenas à instrução primária, como referido em estudo realizado por Candeias ${ }^{5}$, seria oportuno incluir, de acordo com Stewien ${ }^{17}$, um programa educativo anti-fumo, em escolas de primeiro grau, através do método correlacionado. Como observa esse autor, tal programa deveria envolver simultaneamente professores, pais e alunos.

Finalmente, dever-se-ia considerar a educação do público em geral. Na Rússia, por exemplo, existem centros locais de educação em saúde que vão além da atuação dos profissionais da saúde através da utilização de indivíduos preparados para desenvolver campanhas anti-fumo. Nos Estados Unidos, os meios de comunicação de massa têm sido amplas e regularmente utilizados. ${ }^{18}$ No que concerne a educação do público, o relatório da União Internacional Contra o Câncer (UICC) apresenta informações detalhadas sobre os objetivos de programas desta natureza, assim como sobre elementos de informação pública que teriam especial interesse para divulgação nos meios de comunicação de massa.

No que respeita ao componente educativo do problema focalizado no presente estudo, poder-se-ia considerar três niveis de intervençāo: (a) conteúdo da mensagem educa- tiva, (h) localização da mensagem educativa e, finalmente, integração da mensagem educativa.

Quanto ao conteúdo da mensagem edutiva, tendo em vista o desconhecimento das entrevistadas no que se refere aos efeitos do fumo para a saúde da mãe e da criança, caberia à educação em saúde caracterizar o binômio materno-fetal como um todo que não pode ser dissociado. Dentro desta perspectiva, cumpriria ressaltar que tudo que prejudica a saúde da mãe, prejudica paralelamente a saúde do feto.

Seria aconselhável incluir, no conteúdo programático, informações a respeito de como proceder para modificar um hábito dessa natureza. Esta sugestāo torna-se oportuna na medida em que de acordo com o documento da OMS ${ }^{19}$, apesar de muitos fumantes desejarem abandonar o hábito do tabagismo, nem por isso conseguem fazê-lo, necessitando, portanto, de instruções e cuidados especiais.

Quanto à localização da mensagem edutiva, de acordo com os dados apresentados, a maioria das entrevistadas não recebeu orientação sobre os efeitos do fumo para o binômio mãe-criança. Caberia à educação, neste caso, identificar em que circunstâncias $\mathrm{e}$, mais especificamente, em que ocasião se deveriam transmitir informações sistemáticas, orais e por escrito, assim como planejar tecnicamente o conteúdo e a veiculação das mesmas.

Finalmente, no que respeita à integraçâo da mensagem educativa, caberia ̀̀ educação em saúde criar condições para que aquela fosse veiculada, de maneira regular e uniforme, pelos elementos da equipe de saúde e pessoal auxiliar, a fim de que todos, e não apenas um ou outro isolada e acidentalmente, se referissem aos riscos decorrentes do hábito de fumar durante a gestação. Esta ação poderia ser benéfica para os próprios veiculadores da mensagem, muitas vezes fumantes eles próprios. 
CANDEIAS, N. M. F. Fumo durante a gestação: aspectos educativos de um problema comportamental. Rev. Saúde públ., S. Paulo, 13:244-53, 1979.

Tornar-se oportuno reiterar, tal como assinala Candeias" que, ao se tentar modificar o comportamento do individuo é preciso considerar, segundo os pressupostos teóricos do enfoque de consistência contingente, não apenas suas atitudes em relação a determinado objeto da saúde pública, mas também em relação à situação em que o mesmo se encontra. Se as gestantes observarem que o médico, a enfermeira e outros elementos ligados ao atendimento médico, têm o hábito do tabagismo, dificilmente se convencerão de que o fumo afeta a saúde materno-infantil.

O objetivo da educação em saúde seria, assim, o de conseguir apoio total por parte dos profissionais e pessoal da saúde com vistas a legitimar a informação transmitida. Além disto, mobilizá-los para que, cada um, individualmente, aceitasse o desafio de provocar, a curto prazo, uma mudança de comportamento no grupo mais imediatamente afetado pelo fumo, ou seja, o grupo das gestantes.

\section{CONCLUSOEES}

1. Das mulheres entrevistadas, $27,9 \%$ fumavam, 4,5\% haviam deixado de fumar e $6,7 \%$ não fumavam.

2. Em relação a conhecimentos relativos aos efeitos do fumo para a unidade materno-fetal, $32,3 \%$ responderam incorretamente: $8,8 \%$ achavam que fumar não afetava nem a mãe e nem a criança; $4,5 \%$ achavam que o fumo atetava apenas a mãe e $19,0 \%$ que afetava apenas a criança.

3. No que respeita à orientação recebida, em todas as faixas etárias, com exceção do grupo com 21 a 24 anos, recairam os valores modais sobre mulheres que não receberam nenhuma orientação sobre os efeitos do fumo para a saúde materno-fetal.

\section{AGRADECIMENTOS}

Ao Dr. Antonio Pedro Mirra, coordenador executivo do Registro de Câncer de São Paulo, pelas sugestões apresentadas.

CANDEIAS, N. M. F. ISmoking during pregnancy: the educational aspects of a behavior problem.] Rev. Saúde públ., S. Paulo, 13:244-53, 1979.

ABSTRACT: The smoking habits of women hospitalized in the obstetrics ward of a general hospital in the city of $S$. Paulo are described as well as the patients' knowledge about the effects of smoking during pregnancy and the orientation received during this period. Some guidelines for health education are presented and the different levels of educational programs on smoking are considered.

UNITERMS: Smoking. Pregnancy. Health education programs. 
CANDEIAS. N M. F. Fumo durante a gestą̧a o aspectos educatros de um moblema compoltamental. Rel. Suide publ., S. Paulo, 13:244-53. 1979.

\section{REFERENCIAS BIBLIOGRAFICAS}

1 BARIC. L et al A study of health education aspects of smoking $m$ pregnaney, Int. J. Hith Ealc., 19(Suppl. 2):1-17, 1976 .

2. BLTLER. N. R. \& ALBERMAN, E. D. Permatal problems. the second report of the 1959 British Permatal Mortality Sitrey. London. E. \& Livingstone. 1969.

3 EUTLER, i. R. \& GOLDSTEIN, H. Smokng in pregnancy and subse. quent child development Bret meri. $\therefore 4 \cdot 573-5,197 \%$

4. BUtLER. N. $R$, et al. Cogarrette smoking in pregnancy: its influence on birth weight and pernatal mortality. Birt. med. J., 1.127-37. 1972.

5. CANDEIAS. N. M F. Educaçü en suade na prevença do risco gravidico prénatal e interconcepcional. São Paulo. 1979. [Tese de Doutoramento - Fuculdade de Saúde Públyca da USP]

b Candetas. N. M. F. Diagnóstico em educação em saúde: un mocelo para análise das relaçóes entre atitudes $e$ práticas na área da saúde. Rer. Sañdr públ, S. Paulo, 13:63-8. 1979

7. COLE. P. V. et al. Smoking durng pregnancy and its effects on the foetus. J. Obstet. Gynaec. Brit. Cwith, ;9:782-7. 1972 .

8. DAVIES. D. P. et al, Cigarrette smoking in pregnancy. associations with maternal weight gain and fetal growth. Lancet, 21:385-i. 1976 .

9. DUFFUS, G, M. \& MACGILLIVRAY, I The incidence of pre-eclamptic toxaemia in smokers and non-smokers. Lancet, $11: 994.1968$.

10. GOLdSTEIN. $H$. et al. Cigarrette smoking in pregnancy and prematurity $P u b l$. Hith Rep, $78: 55: 2-61.1964$.
11. GRAY, X ed Lieng crincer prevention. wadelines for smokmy control. Ge nera. International Union Against cancer. 1977, IUICC Techn. Pep ser. 28).

12. HARDY, J. B. \& MELLits, E. D. Dues maternal smoking during presnancy Lave a long-term effect on the child: Lancet, 23:1332-6. 1972.

1\%. HERRIOT, A et al Charrette smokne in presnancy, Lancet, 1 771-3. 1962.

14. LOWE. C. R. Effect of mother's smoking habits on birth weight of their children, Brt. med. J., 2673-6. 1959.

15. MCGARAY, J. M. \& ANDREWS, J. A. Smoking in pregnancy and vitamm $\mathrm{B}_{1: 2}$ metaholism Brt. med. J., $274-\overline{\mathrm{r}}$. 1972 .

16. SIAIPSON. W. Y. A preliminary report on clgarrette. Smoking and the moidenca of prematurity Amer. J. Obstet. Gynec, $73 \cdot 808-15,1957$.

$\therefore$ STEWIEN. G T. de M. O adnlescente $\rho$ fumo. São Paulo, 1977. [Dissertação de Mestrado - Faculdade de Saude Públice da USP]

13. U. S. PUBLiC HEalth SERvice. Fiogress against cancer: 1970. apud SARALEGUI FADRON. J. Tabaquismo y educación. Montevideo. Mmlster'o de Salud Publica. 1977.

19. WORLD HEALTH ORGANIZATION. Expert Committee on Smoking and its Effects on Health. Geneva. 1974 Smoke and its effects on health: report Genera, 1975. (Tech. Rep. Ser.. 568).

Recebido para publicacâo em of 06/197a

Aprotado para publicaço em 19 :06/1979 